\title{
Humo de tabaco en vehículos: medición de partículas de materia 2,5 micras
}

\author{
Antonella Pippo*, Laura Llambí*, Carolina Parodi ${ }^{*}$, Mary Barros†, Virginia Núñez, \\ Mauricio Minacapilli*, Mercedes Colomar ${ }^{\ddagger}$, Álvaro Ciganda ${ }^{\ddagger}$, Fiorella Cavalleri§, \\ Juan José Goyeneche
}

\section{Resumen}

Introducción: proteger a la población del humo de segunda mano (HSM) es uno de los principios de la Organización Mundial de la Salud en el marco del control del tabaco. Existen pocos datos acerca de la exposición de HSM en vehículos en América del Sur. Este estudio tuvo como objetivo determinar el nivel de dicha exposición.

Materiales y método: se midieron niveles de micropartículas de materia de 2,5 micras de diámetro $\left(\mathrm{PM}_{2,5}\right)$ que vehiculizan el HSM en la vía aérea, en modelos expertimentales en autos de fumadores y no fumadores.

Resultados: la media de la concentración de $\mathrm{PM}_{2,5}$ fue de $181 \mu \mathrm{g} / \mathrm{m}^{3}$ en los autos de fumadores y de $0 \mu \mathrm{g} / \mathrm{m}^{3}$ en los autos de no fumadores $(p<0,001)$. La máxima concentración fue de $2.900 \mu \mathrm{g} / \mathrm{m}^{3}$ en un auto de fumador estacionado con la ventanilla del conductor parcialmente abierta.

Conclusiones: las concentraciones de $\mathrm{PM}_{2,5}$ en vehículos en los que se fuma alcanzó niveles altos, similares a los que se encuentran en ciertos países con políticas de control de tabaco débiles. Este hecho determina la necesidad de nuevas políticas públicas para eliminar el HSM de los vehículos para proteger la salud pública.

Palabras clave: Humo de segunda mano

Prevención

Salud pública

Key words: $\quad$ Secondhand smoke

Prevention

Public health

\footnotetext{
* Unidad de Tabaquismo, Clínica Médica "A" Hospital de Clínicas, Facultad de Medicina, Universidad de la República, Uruguay. † Unidad de Tabaquismo, Dpto. de Psicología Médica, Hospital de Clínicas, Facultad de Medicina, Universidad de la República, Uruguay. ¥ Unidad de Investigación Clínica y Epidemiológica Montevideo, UNICEM, Montevideo, Uruguay.

$\S$ Dpto. de Métodos Cuantitativos. Facultad de Medicina, Universidad de la República, Uruguay.

II Instituto de Estadística, Facultad de Ciencias Económicas y Administración, Universidad de la República. Uruguay.

** Dpto. de Medicina Preventiva y Social. Facultad de Medicina, Universidad de la República, Uruguay.

Unidad de Tabaquismo.

Clínica Médica "A" y Dpto. de Psicología Médica

Hospital de Clínicas, Facultad de Medicina. Universidad de la República.

Correspondencia: Dra. Antonella Pippo. Hospital de Clínicas. Avda. Italia s/n esq. Las Heras, piso 11. CP11600. Montevideo, Uruguay.

Correo electrónico: antonellapippolefosse@gmail.com

Este estudio fue financiado por los fondos concursables del Programa de Apoyo a la Investigación de la Comisión Honoraria de Lucha contra el

Cáncer - Fundación Manuel Pérez.

Los autores declaran no poseer conflictos de interés.

Recibido: $3 / 6 / 18$

Aprobado: 1/10/18
} 


\section{Introducción}

El tabaquismo es la principal causa de enfermedad y muerte evitable en el mundo, constituyendo la mayor pandemia del siglo XX. De no modificarse los patrones de consumo actual, continuará siéndolo durante el siglo $\mathrm{XXI}^{(1,2)}$. Para controlar el consumo de tabaco se aprueba en 2003 el Convenio Marco de la Organización Mundial de la Salud (OMS) para el Control del Tabaco (CMCT). Lograr ambientes 100\% libres de humo de tabaco es una de las seis áreas focales de la estrategia MPOWER (por su sigla en inglés, Monitor Protect Offer Warn Enforce Raise) de dicho tratado ${ }^{(3,4)}$. Uruguay ratificó dicho tratado convirtiéndose en 2006 en el primer país libre de humo de las Américas.

El humo de tabaco contiene más de 7.000 sustancias tóxicas, entre las cuales se encuentran aproximadamente 69 carcinógenos ${ }^{(2)}$. Estas sustancias son vehiculizadas a través de micropartículas de materia de diámetro menor a 2,5 micras $\left(\mathrm{PM}_{2,5}\right)$ que penetran profundamente en la vía respiratoria llegando a los alvéolos. Estas forman parte de un conjunto de partículas orgánicas e inorgánicas que se clasifican según su diámetro y provienen en su mayoría de la combustión ${ }^{(9)}$. Respirar cualquier cantidad de humo de segunda mano (HSM) se asocia con un daño a la salud ${ }^{(10)}$. Existe evidencia suficiente que muestra una relación causal entre la exposición pasiva al humo de tabaco y el cáncer de pulmón, de mama, de la esfera otorrinolaringológica, el accidente cerebrovascular, la enfermedad cardiovascular y síntomas respiratorios crónicos $^{(2,11)}$.

La OMS alerta que ni la división de ambientes para fumadores y no fumadores, ni la ventilación, protegen a los no fumadores del daño. La única forma efectiva de proteger a las personas de esta exposición es la implementación de ambientes 100\% libres de humo de taba$\mathrm{co}^{(1)}$.

Actualmente mueren 5 millones de fumadores por año y 600.000 fumadores pasivos debido a la exposición al HSM. Debido a la política de control de tabaco, en Uruguay la prevalencia de consumo de tabaco ha tenido un descenso sostenido siendo en la actualidad $21,1 \%$ en adultos ${ }^{(5-7)}$. No obstante, la carga de enfermedad atribuible al tabaco alcanza las 6.542 muertes anuales $(68 \%$ hombres y $32 \%$ mujeres) y determina 135.622 años de vida saludable perdidos. El $8 \%$ de dichas muertes ocurre en fumadores pasivos, o sea en no fumadores expuestos al humo de tabaco ajeno ${ }^{(8)}$.

Si bien en nuestro país descendió la exposición al HSM de acuerdo a la Encuesta Continua de Hogares, en 2011 en 22,8\% de los hogares se fumaba y en 2014 bajó a 18,5\%. En los hogares con niños menores de 5 años este porcentaje fue de $16,4 \%{ }^{(6)}$.
Según una publicación del año 2014, 81\% de los fumadores que tienen vehículo no permiten que se fume en el interior, y $88 \%$ de los fumadores apoyaría una ley que prohíba fumar en los vehículos ${ }^{(12)}$, similar a lo que reportan países con políticas de control de tabaco ${ }^{(13)}$.

De acuerdo a un estudio reciente de nuestro equipo, en Uruguay, se estima que en $12 \%$ a $19 \%$ de los vehículos se fuma, y en un tercio existe otro individuo expuesto a HSM $^{(15)}$. El reconocimiento de este problema y la obtención de datos objetivos sobre los niveles de concentración de HSM en autos servirán para impulsar la adopción de nuevas medidas de control de tabaco y a la autorregulación de la población en el cuidado de su salud, confiriéndole a este estudio relevancia tanto científica como social.

El objetivo de este estudio fue medir los niveles de exposición de $\mathrm{PM}_{2,5}$ en vehículos de fumadores.

\section{Materiales y método}

\section{Diseño del estudio}

Estudio observacional analítico, de corte transversal.

\section{Metodología}

Este estudio consistió en la medición de HSM a través de la concentración de $\mathrm{PM}_{2,5}$ en diferentes modelos experimentales de ventilación y de circulación de vehículos.

\section{Medición de $P M_{2,5}$}

Se midieron las $\mathrm{PM}_{2,5}$ en seis modelos experimentales: en movimiento y estacionados y con diferentes grados de ventilación. Se reclutaron voluntarios fumadores que fumaran habitualmente en sus vehículos para realizar las mediciones, y voluntarios no fumadores que tuvieran vehículos donde no se fumara.

Las $\mathrm{PM}_{2,5}$ se midieron con monitores portátiles SidePack ${ }^{\circledR}$-TSI. Se utilizó el modelo AM510, que mide por fotometría láser las partículas de materia en $\mu \mathrm{g} / \mathrm{m}^{3}$. Cada medición duró al menos 30 minutos. Este tiempo fue considerado en base a nuestro interés en realizar las mediciones en la ciudad y el tiempo estándar aproximado de traslado dentro de la misma, además nos inspiramos en protocolos similares internacionales ${ }^{(16)}$ y en nuestro interés en comparar las concentraciones de micropartículas encontradas en nuestro estudio con las realizadas en nuestro país en otros espacios cerrados, donde se tomó como tiempo de medición 30 minutos ${ }^{(17)}$. Se registró a qué modelo experimental correspondía cada medición y la información almacenada en el monitor se descargó al software informático TrakPro ${ }^{\circledR}$ para su análisis (figura 1). 


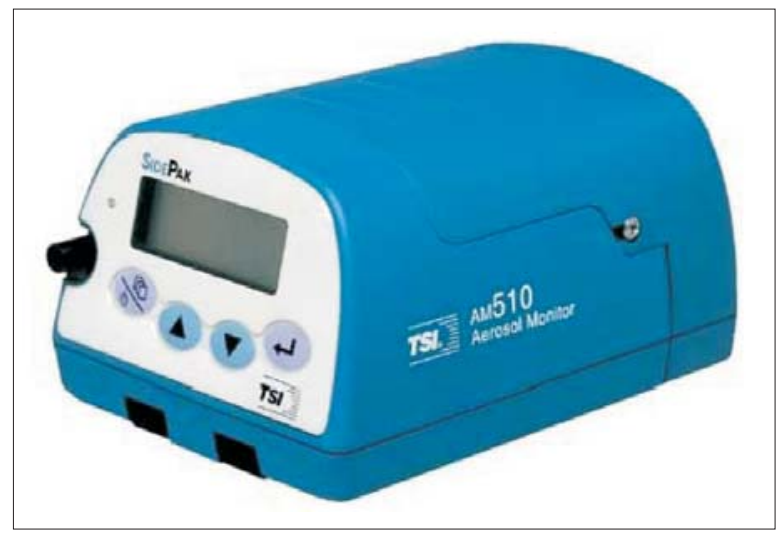

Figura 1. Monitor medidor de partículas SidePack ${ }^{\circledR}$ -TSI. Modelo AM510.

Los autos en movimiento circularon a una velocidad de $45 \mathrm{~km} /$ hora. Cada modelo se midió en cuatro vehículos de fumadores y en dos vehículos de no fumadores como control, totalizando 36 mediciones. Los modelos experimentales fueron:

- Modelo 1: auto en movimiento con ventanilla adyacente al fumador abierta.

- Modelo 2: auto en movimiento con ventanilla adyacente al fumador semiabierta (menos del $50 \%$ abierta).

- Modelo 3: auto en movimiento con más de una ventanilla abierta o semiabierta.

- Modelo 4: auto estacionado con ventanilla adyacente al fumador abierta.

- Modelo 5: auto estacionado con ventanilla adyacente al fumador semiabierta (menos del 50\% abierta).

- Modelo 6: se realizó una medición denominada residual, que consistió en medir las $\mathrm{PM}_{2,5}$ tres horas luego de que se hubiera fumado en un auto estacionado y cerrado, independientemente de las condiciones de ventilación o movimiento en las que se había fumado.

\section{Análisis estadístico}

Los niveles de $\mathrm{PM}_{2,5}$ se reportan en medianas y rango intercuartílico debido a que no presentaron una distribución normal. Se utilizó la prueba de Mann Whitney y de Wilcoxon para comparar los diferentes modelos medidos. Se utilizó el test de chi ${ }^{2}$ para comparación de proporciones. Se utilizó el software estadístico SPSS versión 20 (IBM Corp., Armonk, NY, USA). Valores de $\mathrm{p}$ $<0,05$ fueron considerados estadísticamente significativos.

\section{Consideraciones éticas}

El protocolo fue aprobado por el Comité de Ética de la Investigación del Hospital de Clínicas, Universidad de la República. Se obtuvo el consentimiento informado de los voluntarios fumadores que participaron en la medición de $\mathrm{PM}_{2,5}$. Se cumplió con las disposiciones de protección de los datos personales y confidencialidad. A los fumadores voluntarios que participaron se les ofreció realizar tratamiento para dejar de fumar en la Unidad de Cesación de Tabaquismo del Hospital de Clínicas en forma gratuita.

El estudio adhirió a las disposiciones del Decreto 379/008 del Ministerio de Salud Pública referente a investigación con seres humanos, y a la Declaración de Helsinki (versión año 2000).

\section{Resultados}

\section{Medición de partículas de materia $P M_{2,5}$}

Las medianas de concentración de $\mathrm{PM}_{2,5}$ en los autos de fumadores considerados en los modelos 1 a 5 fue 181 $\mu \mathrm{g} / \mathrm{m}^{3}$ rango intercuartílico (RIQ) $\left(65-501 \mu \mathrm{g} / \mathrm{m}^{3}\right)$ mientras que en los no fumadores fue $0 \mu \mathrm{g} / \mathrm{m}^{3}$ RIQ ( $0-1$ $\left.\mu \mathrm{g} / \mathrm{m}^{3}\right)$, encontrándose una diferencia estadísticamente significativa $(\mathrm{p}<0,001)$ (tabla 1$)$.

La concentración máxima encontrada fue de 2.900 $\mu \mathrm{g} / \mathrm{m}^{3}$ en el modelo de auto estacionado con ventanilla parcialmente abierta. Cuando se estimó la concentración de materia particulada para un período de ocho horas (expresadas en medias ponderadas por tiempo), también se mantuvo una diferencia estadísticamente significativa entre los autos con fumadores $54 \mu \mathrm{g} / \mathrm{m}^{3}$ y aquellos de no fumadores $0 \mu \mathrm{g} / \mathrm{m}^{3}(\mathrm{p}<0,001)$.

Las concentraciones de $\mathrm{PM}_{2,5}$ en autos de fumadores fueron mayores con el modelo de auto estacionado respecto al modelo de auto en movimiento.

Se observaron variaciones en las concentraciones de $\mathrm{PM}_{2,5}$ de acuerdo a la apertura de las ventanillas, pero las diferencias no fueron estadísticamente significativas (tabla 1).

Se detectó humo de tabaco en los autos, aún tres horas después de haber fumado, alcanzando una mediana de concentración de $\mathrm{PM}_{2,5}$ de $5 \mu \mathrm{g} / \mathrm{m}^{3}$ (RIQ 2-8 $\mu \mathrm{g} / \mathrm{m}^{3}$ ).

\section{Discusión}

\section{Hallazgos principales}

La concentración de $\mathrm{PM}_{2,5}$ en los autos en los que se fumó tuvo una mediana de $181 \mu \mathrm{g} / \mathrm{m}^{3}$ y una concentración máxima de $2.900 \mu \mathrm{g} / \mathrm{m}^{3}$, observándose que el movimiento del vehículo y el estado de apertura de las ventanillas afecta la concentración de las partículas, pero no en forma estadísticamente significativa. 


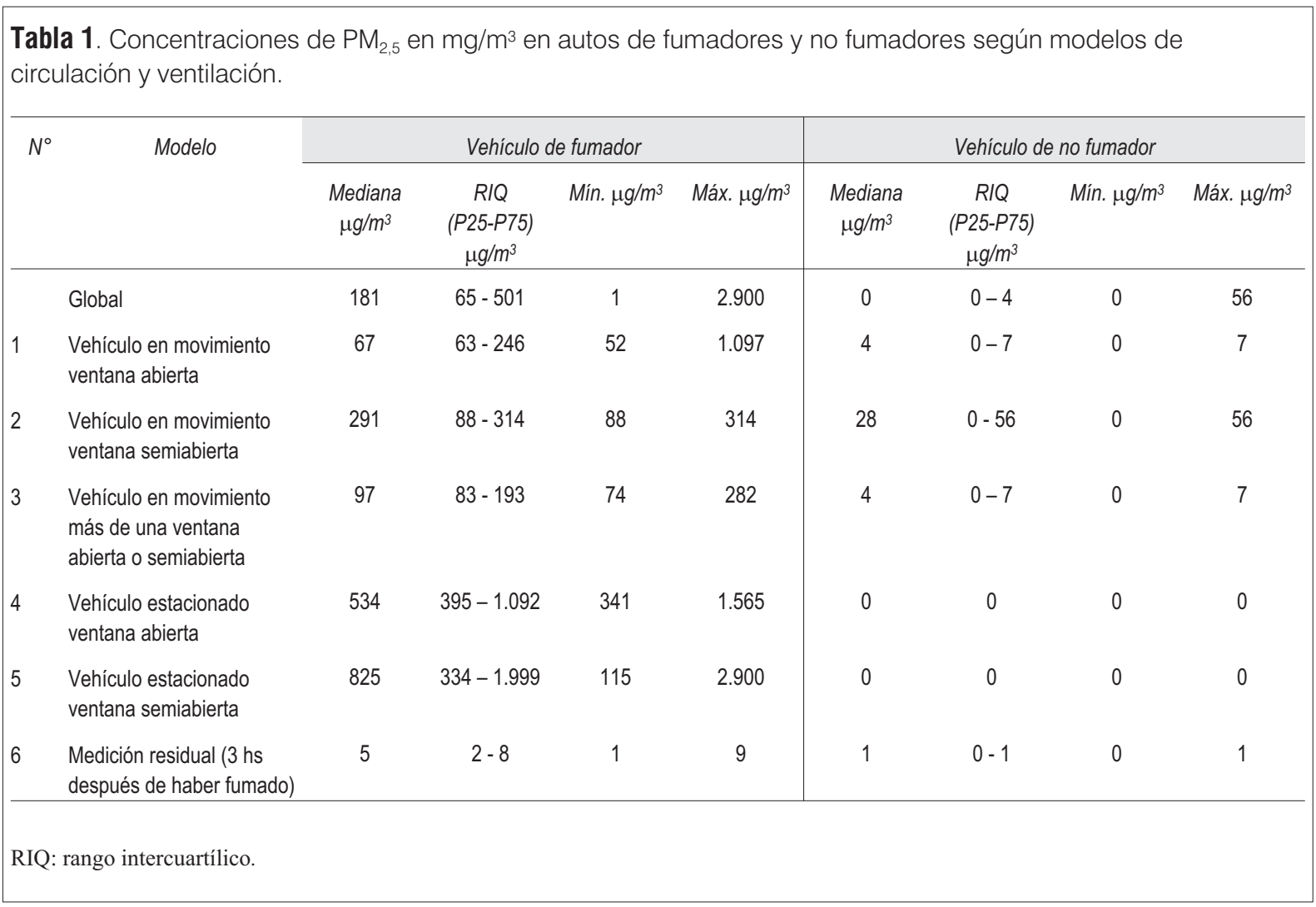

\section{En contexto}

La concentración de partículas de materia encontrada en nuestro estudio fue elevada. No existe un umbral de seguridad o punto de corte establecido por debajo del cual se pueda asegurar que la exposición no sea dañina para la salud, habiéndose demostrado efectos adversos con concentraciones de 3 a $5 \mu \mathrm{g} / \mathrm{m}^{3}$ tanto en exposiciones breves como prolongadas ${ }^{(18)}$. La Guía de Calidad del Aire de la OMS establece un punto de corte de seguridad de $25 \mu \mathrm{g} / \mathrm{m}^{3(19)}$. En 2008, Hyland y colaboradores publicaron un estudio en 32 países, incluido Uruguay, en el que se midió la concentración de partículas $\mathrm{PM}_{2,5}$ en ambientes cerrados de bares, medios de transporte y hoteles. En Uruguay se encontró una media de $17 \mu \mathrm{g} / \mathrm{m}^{3}$ en los casos en los que no se habían observado personas fumando durante la medición y $33 \mu \mathrm{g} / \mathrm{m}^{3}$ en los casos en los que sí se habían observado personas fumando ${ }^{(17)}$. Por lo tanto, la concentración encontrada en nuestro estudio es 5 a 10 veces mayor a la reportada en otros ambientes cerrados en nuestro país desde que entró en vigencia la normativa de ambientes libre de humo de tabaco. En dicho estudio, cuando se analizan las concentraciones de $\mathrm{PM}_{2,5}$ discriminando por tipo de locación, la más elevada es la que se encontró en bares en los que se permite fumar, alcanzando una media de $303 \mu \mathrm{g} / \mathrm{m}^{3(19)}$.
Por lo tanto, se puede afirmar que las concentraciones encontradas en nuestro estudio en autos estacionados son mayores a las observadas en bares de países sin políticas de control de tabaco, aun con una o más ventanillas abiertas. En los casos en que el vehículo estaba estacionado y con la ventanilla solo parcialmente abierta, el valor hallado en nuestro estudio fue entre 25 y 48 veces el reportado en nuestro país en el estudio de Hyland, sugiriendo que en un ambiente pequeño como es el de un auto, la materia particulada alcanza rápidamente concentraciones muy elevadas ${ }^{(17)}$. La concentración encontrada es consistente con lo reportado por otros estudios de similar metodología así como las variaciones de la misma de acuerdo al grado de movimiento y ventilación del vehícu$10^{(16,19-23)}$. El valor que encontramos en vehículos donde no se fumó es despreciable, salvo en uno de los modelos en que el valor encontrado pudo deberse a otra fuente de $\mathrm{PM}_{2,5}$, como otras fuentes de combustión, polución ambiental, o pasaje de un fumador cerca del vehículo durante el período de medición. No obstante, el valor máximo hallado en vehículos en los que se fumó es 50 veces mayor al máximo encontrado en controles. Jones y colaboradores estudiaron las concentraciones de diferentes biomarcadores de consumo de tabaco, como cotinina en plasma y orina, y además dosificaron 4-metilnitrosamino-1(3-piridil)-1-butanol (NNAL), un metabolito de un 
carcinógeno pulmonar específico en no fumadores expuestos a HSM en autos. En dicho estudio se reportaron incrementos significativos de estos biomarcadores bajo exposición a $\mathrm{PM}_{2,5}$ a similares concentraciones que las encontradas en nuestro estudio, destacándose el incremento de nitrosamina NNAL en 27 veces respecto al valor basal $^{(24)}$.

En nuestro estudio se encontró una concentración de HSM mayor en autos con más de una ventanilla abierta que en los que tenían solo una abierta, si bien los RIQ se superponen y la concentración máxima alcanzada es mayor en la segunda situación. Esta discordancia también fue encontrada por Edwards y colaboradores ${ }^{(25)}$, cuando el cigarrillo es mantenido fuera del auto entre las pitadas. Si bien se esperaría que la concentración fuese menor a mayor ventilación, otros variables pueden incidir. Una revisión sistemática realizada por Raoof y colaboradores en 2015 que incluyó 12 estudios con mediciones cuantitativas de HSM en autos, encontró un rango de concentraciones de $\mathrm{PM}_{2,5}$ de hasta 13.150 en casos de vehículos con todas las ventanillas cerradas, concluyendo que no solo el movimiento y el estado de ventanillas influye en las concentraciones de $\mathrm{PM}_{2,5}$ como en nuestro estudio, sino otras variables tales como velocidad y uso de aire acondicionado. Dichos autores concluyen que ningún sistema de ventilación logra eliminar el $\mathrm{HSM}^{(22)}$.

\section{Fortalezas y debilidades}

Las mediciones de $\mathrm{PM}_{2,5}$ siguieron un protocolo estandarizado, pero pudieron tener variaciones entre las mismas por diferencias en las condiciones climáticas, modelo y tamaño del vehículo, o la velocidad exacta a la que circulaba. Por este motivo cada modelo fue medido en varias instancias y en su correspondiente control no fumador.

\section{Conclusiones e implicancias}

Las concentraciones del HSM en vehículos, medidas en materia particulada $\mathrm{PM}_{2,5}$ son elevadas, similares a las encontradas en bares de países sin políticas de control de tabaco en salud pública. Este estudio aporta evidencia que puede ser utilizada por tomadores de decisión en política sanitaria, en un país con una marcada política de control de tabaco que ha mostrado resultados beneficiosos para la salud de la población. Así mismo, las regulaciones han tenido amplio respaldo por parte de la sociedad civil. Nuevas regulaciones sobre fumar en autos, sobre todo en presencia de terceros y particularmente de niños, como se han adoptado en otros países, serían una estrategia fundamental de protección a la salud de no fumadores y fumadores, y contribuirán a la motivación para el cese del consumo en estos últimos.

Considerando los niveles de acatamiento observados en la población del país, que manifiestan un cambio cultural sobre la exposición al HSM, es recomendable también la difusión de esta información con fines de promoción y prevención.

Si bien nuestro estudio no abordó el tema del humo de tercera mano en autos, los niveles de particulado encontrados podrían hacer sospechar la alta contaminación de tapizados, techos y tableros en los autos de fumadores. Es un área poco investigada globalmente, no existen estudios en nuestro medio, en la que se debería profundizar dado el creciente interés en el tema por las posibles implicancias en la salud especialmente de niños ${ }^{(26,27)}$.

\section{Agradecimientos}

A la Dra. Ana Navas, Ancien PhD de la Escuela de Salud Pública de la Universidad de Johns Hopkins, quien generosamente nos prestó los monitores SidePack de medición de partículas de humo de segunda mano.

A los ingenieros químicos Andrea De Nigris y Pablo Franco de la Unidad de Calidad de Aire de la Intendencia de Montevideo por su asesoramiento. A los voluntarios que aceptaron participar en este estudio.

\section{Abstract}

Introduction: Protection from second-hand smoke (SHS) is one of the main principles of the World Health Organization Framework Convention for Tobacco Control. Limited data is available on SHS exposure in vehicles in South America. This study aimed to assess the levels of exposure.

Methods: Levels of respirable and fine suspended particles with 2.5 micrometres or less $\left(\mathrm{PM}_{2.5}\right)$ diameter were measured in different models in smokers' and non-smoker's vehicles.

Results: Median $\mathrm{PM}_{2.5}$ concentration was $181 \mu \mathrm{g} / \mathrm{m}^{3}$ in "smoking vehicles" and $0 \mu \mathrm{g} / \mathrm{m}^{3}$ in "non-smoking vehicles" $(p<0.001)$. The highest concentration reached $2.900 \mu \mathrm{g} / \mathrm{m}^{3}$ in a parked car with the driver's window partially open.

Conclusions: Concentration of $\mathrm{PM}_{2.5}$ in vehicles reached high levels, similar to those at certain sites in countries with weak tobacco control policies. These facts underscore a need for new public policies to eliminate SHS in vehicles to protect public health.

\section{Resumo}

Introdução: um dos princípios da Organização Mundial da Saúde no contexto do controle do tabaquismo é proteger a população da fumaça de segunda-mão (HSM). Existem poucos dados sobre a exposição de HSM em veículos na América do Sul. O objetivo deste estudo foi identificar um mecanismo para determinar o nível desta exposição. 
Materiais e métodos: utilizando modelos experimentais em veículos de fumantes e não fumantes foram medidos os níveis de micropartículas de matéria de 2,5 micras de diâmetro $\left(\mathrm{PM}_{2,5}\right)$ transportados pela HSM na via aérea.

Resultados: á concentração média de $\mathrm{PM}_{2,5}$ foi 181 $\mu \mathrm{g} / \mathrm{m}^{3}$ nos automóveis de fumantes e $0 \mu \mathrm{g} / \mathrm{m}^{3}$ nos automóveis de não fumantes $(\mathrm{p}<0.001)$. A concentração máxima de $2.900 \mu \mathrm{g} / \mathrm{m}^{3}$ foi encontrada no automóvel estacionado de um fumante com a janela do motorista parcialmente aberta.

Conclusões: as concentrações de $\mathrm{PM}_{2,5}$ em veículos de fumantes alcançou níveis altos, similares aos encontrados em alguns países com políticas de controle de tabaco débeis. Este fato determina a necessidade de novas políticas públicas para eliminar a HSM dos veículos para proteger a saúde pública.

\section{Bibliografía}

1. World Health Organization. WHO Report on the Global Tobacco Epidemic. 2013.

2. Eriksen M, Mackay J, Schluger N, Gomeshtapeh FI, Drope J. The Tobacco Atlas. 5th ed. American Cancer Society, editor. Atlanta; 2015.

3. World Health Organization. WHO report on the global tobacco epidemic: 2008-the MPOWER package. 2008.

4. World Health Organization. Framework Convention on Tobacco Control (FCTC). Geneva; 2004.

5. Abascal W, Esteves E, Goja B, González Mora F, Lorenzo A, Sica A, et al. Tobacco control campaign in Uruguay: a population-based trend analysis. Lancet. 2012 3;380(9853): 1575-82.

6. Instituto Nacional de Estadística Uruguay. Encuesta Continua de Hogares. 2014.

7. Junta Nacional de Drogas Uruguay. $6^{\circ}$ Encuesta Nacional sobre Consumo de Drogas en Estudiantes ed Enseñanza Media. 2014.

8. Ministerio de Salud Publica Uruguay. Programa de Enfermedades Crónicas No Transmisibles. Estudio de Carga Atribuible al Consumo de Tabaco. 2014

9. Nerín I, Alayeto C, Córdoba R, López MJ, Nebot M. Measurement of Fine Breathable Particles $\left(\mathrm{PM}_{2.5}\right)$ as a Marker of Environmental Smoke in Catering Establishments in Zaragoza. Arch Bronconeumol (English Version). Elsevier; 2011 1;47(04):190-4.

10. Comisión Honoraria de Lucha contra el Cancer. Hogares Libres de humo de tabaco: por la salud de nuestros niños y niñas. 2009.

11. Centre for Disease Control and Prevention (US). The Health Consequences of Involuntary Exposure to Tobacco Smoke A Report of the Surgeon General. 2006;

12. Proyecto ITC (Agosto 2014). Informe National ITC Uruguay. Resultados de los Relevamientos 1 a 4 de la Encuesta (2006-12). Universidad de Waterloo, Waterloo, Ontario, Ca- nadá; Centro de Investigación para la Epidemia del Tabaquismo y Universidad de la República, Uruguay.

13. Gendall P, Hoek J, Maubach N, Edwards R. Public support for more action on smoking. New Zeal Med J. New Zealand Medical Association (NZMA); 2013;126(1375).

14. Jones MR, Navas-Acien A, Yuan J, Breysse PN. Secondhand tobacco smoke concentrations in motor vehicles: a pilot study. Tob Control. 2009;18(5):399-404

15. Llambi L, Barros M, Parodi P, Pippo A, Nuñez V, Colomar $\mathbf{C}$ et al. Prevalence of invehicle smoking and secondhand smoke exposure in Uruguay. Tob Control. 2018;0:1-3

16. Sendzik T, Fong GT, Travers MJ, Hyland A. An experimental investigation of tobacco smoke pollution in cars. Nicotine Tob Res. 2009 ;11(6):627-34.

17. Hyland a, Travers MJ, Dresler C, Higbee C, Cummings KM. A 32-country comparison of tobacco smoke derived particle levels in indoor public places. Tob Control. 2008;17(3):159-65.

18. World Health Organization. Guías de calidad del aire de la OMS relativas al material particulado, el ozono, el dióxido de nitrógeno y el dióxido de azufre. 2005.

19. World Health Organization. WHO Guidelines for Indoor Air Quality Guidelines. Geneva; 2010.

20. Liu S, Zhu Y. A case study of exposure to ultrafine particles from secondhand tobacco smoke in an automobile. Indoor Air. $2010 ; 20(5): 412-23$.

21. Northcross AL, Trinh M, Kim J, Jones IA, Meyers MJ, Dempsey DD, et al. Particulate mass and polycyclic aromatic hydrocarbons exposure from secondhand smoke in the back seat of a vehicle. Tob Control. 2014 ;23(1):14-20.

22. Raoof S, Agaku IT, Vardavas CI. A systematic review of secondhand smoke exposure in a car: Attributable changes in atmospheric and biological markers. Chron Respir Dis. 2015;12:120-131. doi:10.1177/1479972315575202.

23. Semple S, Apsley A, Galea KS, MacCalman L, Friel B, Snelgrove V. Secondhand smoke in cars: assessing children's potential exposure during typical journey conditions. Tob Control. 2012;21(6):578-583. doi:10.1136/tobaccocontrol-2011-050197.

24. Jones I a, St Helen G, Meyers MJ, Dempsey D a, Havel C, Jacob P, et al. Biomarkers of secondhand smoke exposure in automobiles. Tob Control. 2014;23(1):51-7.

25. Edwards R, Wilson N, Pierse N. Highly hazardous air quality associated with smoking in cars: New Zealand pilot study. N Z Med J. 2006;119(1244):U2294

26. Matt GE, Quintana PJE, Hovell MF, et al. Residual tobacco smoke pollution in used cars for sale: air, dust, and surfaces. Nicotine Tob Res. 2008; 10:1467-75. [PubMed: 19023838].

27. Drehmer J, Hipple Walters, Nabi-Burza E, Winickoff J. Guidance for the Clinical Management of Thirdhand Smoke Exposure in the Child Health Care Setting. J Clin Outcomes Manag. 2017; 24(12): 551-559. 